Sabine Blaschke, Martin Menrad ${ }^{*}$

\title{
Einführung und Umsetzung gewerkschaftlicher Frauenquoten: Befunde zu zwei österreichischen Gewerkschaften $^{* *}$
}

\section{Zusammenfassung}

Im vorliegenden Beitrag geht es um die Rekonstruktion der Prozesse der Einführung und Umsetzung der Frauenquote in zwei österreichischen Gewerkschaften: einer ArbeiterInnengewerkschaft des privaten Dienstleistungssektors und einer Angestelltengewerkschaft. Zur Bearbeitung der Fragestellung wurden 41 leitfadengestützte Interviews geführt. In beiden Gewerkschaften erschweren die geschlechtsspezifische innerfamiliale Arbeitsteilung, eine männlich geprägte Organisationskultur und männlicher Widerstand die Implementation der Quotenregelung. In der ArbeiterInnengewerkschaft läuft die Umsetzung weniger gut als in der Angestelltengewerkschaft; vor allem ist es schwieriger, Frauen für die Ausübung von Funktionen zu gewinnen, und es gibt weniger Unterstützung durch männliche Gewerkschafter. Die Einführung der Quote ohne grundlegende Diskussion und die geringere Dichte an Betriebsratskörperschaften in den relevanten Branchen des privaten Dienstleistungsbereichs können als wichtige Ursachen für die Probleme in der ArbeiterInnengewerkschaft identifiziert werden.

\section{Introduction and implementation of women's quotas in trade unions: Findings from two Austrian unions}

\author{
Abstract \\ This article deals with the reconstruction of the processes of introduction and implementation of \\ women's quotas in two Austrian trade unions: a private services blue-collar union and a white-collar \\ union. For investigating this issue 41 guided interviews were conducted. In both unions the gendered \\ division of labour in the family, the male organizational culture and male resistance make the imple- \\ mentation of the quota rule difficult. In the blue-collar union the implementation works less well than \\ * Dr.in Sabine Blaschke, Ao. Universitätsprofessorin an der Universität Wien, Institut für Wirtschaftssoziolo- \\ gie, Oskar-Morgenstern-Platz 1, A-1090 Wien. \\ E-Mail: sabine.blaschke@univie.ac.at (Korrespondenzadresse) \\ Mag. Martin Menrad, Soziologe im Bereich Betriebliche Gesundheitsförderung in der Oberösterreichischen \\ Gebietskrankenkasse. \\ E-Mail: martin.menrad@gmail.com \\ ** Artikel eingegangen: 12.10.2015 \\ revidierte Fassung akzeptiert nach doppelt-blindem Begutachtungsverfahren: 25.5.2016.
}


in the white-collar union; in particular it is more difficult to find women for exercising the mandates and there is less support from male unionists. The introduction of the women's quota without fundamental discussion and the lower density of works councils in the relevant private services industries can be identified as important reasons for implementation problems in the blue-collar union.

Key words: trade unions, women, expert interviews, Austria (JEL: J51, J59)

\section{Einleitung ${ }^{1}$}

Lange Zeit waren Frauen in den Gremien der österreichischen Gewerkschaften nur selten entsprechend ihrem Anteil an den Mitgliedern vertreten (vgl. Blaschke, 2008). In den letzten Jahren hat die Mehrheit der Gewerkschaften entweder für die zentraleren Gremien oder für alle Gremien Frauenquoten eingeführt, wodurch sich die Repräsentation von Frauen in den gewerkschaftlichen Entscheidungsstrukturen wesentlich verbessert hat. Der vorliegende Beitrag untersucht die Einführung und Umsetzung von Frauenquoten in zwei Gewerkschaften: der Gewerkschaft der Privatangestellten, Druck, Journalismus, Papier (GPA-djp, sie organisiert mit wenigen Ausnahmen die nicht-öffentlichen Angestellten aller Branchen) und der Gewerkschaft vida (sie organisiert die ArbeiterInnen im privaten Dienstleistungssektor mit Ausnahme des ehemals staatlichen Post- und Telekombereichs). Befunde aus 41 Interviews in den beiden Gewerkschaften werden präsentiert.

Die Analyse soll zum einen Einblick in die Abläufe der Einführung und Umsetzung der Frauenquote geben. Zum anderen soll sie Aufschlüsse darüber geben, wie sich Prozesse auf der Mikroebene zwischen den beiden Gewerkschaften unterscheiden, und mehreren Annahmen dazu nachgehen. Denn Befunde einer früheren Untersuchung haben ergeben, dass in Österreich und Deutschland in den ArbeiterInnengewerkschaften des privaten Dienstleistungssektors Frauen in Gremien und Positionen deutlich unterrepräsentiert sind (Blaschke, 2008, 2015). Mittlerweile hat sich in Österreich aufgrund der Einführung der Frauenquote das Ausmaß der Repräsentation der Frauen in den Gremien zwar angeglichen (oder zumindest stark angenähert); es ist jedoch wahrscheinlich, dass sich die beiden Gewerkschaften in Bezug auf die Ausgestaltung der Partizipation von Frauen weiterhin unterscheiden.

Welche Prozesse Unterschiede zwischen Gewerkschaften mit unterschiedlich qualifizierten Mitgliedern zustande bringen, war bisher nicht Gegenstand von Untersuchungen. Zu den Prozessen auf der Mikroebene, die an der Herstellung von Geschlechterverhältnissen beteiligt sind, gibt es mehrere Arbeiten, sie richten sich auf verschiedene Fragestellungen: Einige Studien widmen sich der Wirkung gewerkschaftlicher Strukturen und Angebote auf die Beteiligung von Frauen (z.B. Colgan \& Ledwith, 2002; Kirton, 2005), andere der Herausbildung einer ,Gender'-Identität bei Gewerkschafterinnen (z.B. Colgan \& Ledwith, 2000; Kirton \& Healy, 2004) oder der Implementation von Vertretungsrechten (z.B. McBride, 2001). Zur Frage systematischer Unterschiede zwischen Gewerkschaften, die von Mit-

1 Wir danken Ingrid Artus und zwei anonymen GutachterInnen für ihre wertvollen Anregungen und hilfreiche Kritik zu einer früheren Fassung dieses Beitrags. Den GesprächspartnerInnen danken wir für ihre Zeit und die Bereitschaft, ihre Erfahrungen mitzuteilen. Das der Arbeit zugrundeliegende Forschungsprojekt wurde durch Fördergelder des Jubiläumsfonds der Oesterreichischen Nationalbank (Projektnummer: 15512) unterstützt. 
gliedern eines bestimmten Qualifikationsniveaus oder Berufsstatus stark dominiert sind, lassen sich in diesen Untersuchungen keine Hinweise finden. Der vorliegende Beitrag trägt zum Füllen dieser Lücke bei. Die Analyse der Einführung und Umsetzung in den beiden Gewerkschaften kann zudem für die Praxis Hinweise darauf geben, welche Probleme bei der Einführung und Umsetzung einer Frauenquote in einer Gewerkschaft auftreten und welche Faktoren zu ihrem Gelingen beitragen können.

\section{Frauenquoten in Gewerkschaften}

Die Unterrepräsentation von Frauen in gewerkschaftlichen Gremien korrespondiert mit der Unterrepräsentation von Frauen in anderen gesellschaftlichen Bereichen: So nehmen erwerbstätige Frauen seltener als Männer Führungspositionen ein und sind Frauen in diversen politischen Ämtern unterrepräsentiert (z.B. Frauenbericht, 2010: 145-146, 360-372). Für die Unterrepräsentation von Frauen in gewerkschaftlichen Gremien und Führungspositionen wurden in diversen Untersuchungen mehrere Gründe identifiziert, welche zu einem großen Teil denen für die Unterrepräsentation von Frauen in anderen Bereichen entsprechen. Auf der einen Seite behindern die männliche Organisationskultur der Gewerkschaften (einschließlich der männlichen Netzwerke) und der Widerstand der männlichen Oligarchien (die Gruppen der zuvor in Führungspositionen Gelangten, die aus Männern bestehen und ihre Macht nicht abgeben wollen) die Partizipation der Frauen (z.B. Healy \& Kirton, 2002; Ledwith, 2012). Auf der anderen Seite streben Frauen von sich aus eine Übernahme von Gewerkschaftsämtern weniger an - wegen des Zeitaufwands, der ihnen neben Berufs- und Versorgungsarbeit nicht machbar erscheint, und aufgrund geringeren Selbstvertrauens (z.B. Blaschke, 2008: 157-260, 248-249; Trebilcock 1991: 411). Auch die geschlechtsspezifische Arbeitsmarktsegregation trägt zur Unterrepräsentation von Frauen bei: Zum einen weil Frauen häufiger geringer qualifizierte Tätigkeiten ausüben und GewerkschaftsfunktionärInnen sich eher aus höher qualifizierten ArbeitnehmerInnen rekrutieren, zum anderen weil Frauen auf wenige Branchen konzentriert sind und dadurch bei einer gleichmäßigen Vertretung von Branchen - wie sie in gewerkschaftlichen Gremien häufig praktiziert wird - seltener vertreten sind (z.B. Trebilcock 1991: 411-412). Schließlich führt der in Gewerkschaften übliche Prozess der Delegation von unten nach oben dazu, dass Frauen (so wie andere Minderheitengruppen) benachteiligt werden und sich eine Unterrepräsentation auf unteren Ebenen nach oben hin verstärkt (z.B. Neyer 1995: 616-617).

Diese Faktoren, die zur Unterrepräsentation von Frauen in gewerkschaftlichen Gremien führen, sind auf der Makro-, Meso- und Mikroebene verortet und fallen somit teilweise unter strukturtheoretische Erklärungen der Geschlechterungleichheit und teilweise unter handlungstheoretische Erklärungen (vgl. Funder, 2002: 294-296). Der Makroebene zugehörig sind die Arbeitsmarktstrukturen und die geschlechtsspezifische ungleiche Verteilung der privaten Versorgungsarbeit, der Mesoebene sind die Organisationsstruktur und -kultur der Gewerkschaften zuzuordnen. Auf der Mikroebene sind die Handlungen angesiedelt, die direkt die Unterrepräsentation von Frauen herstellen. Traditionelle Geschlechtsstereotype bewirken, dass Männer Frauen mit Ablehnung oder Vorbehalten begegnen und Frauen daran zweifeln, ob sie die notwendigen Voraussetzungen für die gewerkschaftliche Arbeit aufwei- 
sen (Trebilcock 1991: 411; vgl. auch Ridgeway \& Correll, 2004: 525-526). Auf verschiedenen Ebenen angesiedelte Faktoren entfalten ihren Effekt teilweise durch das gemeinsame Auftreten oder werden dadurch verstärkt. So wird die hemmende Wirkung der geschlechtsspezifischen Arbeitsteilung in der privaten Versorgungsarbeit durch eine auf von Versorgungsarbeit unbelastete Funktionäre zugeschnittene Organisationskultur verschärft.

Seit den 1970er Jahren werden Quotenregelungen als Mittel zur Aufhebung der Unterrepräsentation von Frauen im beruflichen und politischen Bereich diskutiert. Mittlerweile haben mehrere Parteien Quotenregelungen für die KandidatInnenlisten der Parlamentswahlen eingeführt (vgl. für Österreich Jarosch, 2001: 171-179; international vgl. z.B. die Beiträge in Dahlerup, 2006a) ${ }^{2}$ und auch Gewerkschaften haben Quotenregelungen in ihre Satzungen aufgenommen. In Erwerbsorganisationen gibt es in Österreich Quotenregelungen nur auf freiwilliger Basis. Auf europäischer Ebene wurden in den letzten Jahren gesetzliche Quotenregelungen für Aufsichtsräte börsennotierter Unternehmen diskutiert und in einigen Ländern, wie z.B. Deutschland, auch beschlossen. Einzigartig gilt in Deutschland seit 2001 für Betriebsratskörperschaften eine gesetzliche Geschlechterquote für dasjenige Geschlecht, das in der Belegschaft in der Minderheit ist.

Regelungen für eine Mindestvertretung von Frauen werden nach dem Grad ihrer Verbindlichkeit unterschieden (vgl. Inhetveen, 2002: 15-16; Pfarr \& Bertelsmann 1989: 91-95). Der Begriff „Frauenquote“ bezeichnet heute in der Regel eine verbindliche Regelung. Zahlenvorgaben, deren Erfüllung auf einer freiwilligen Basis erwünscht wird, werden u.a. als „Soll-Regelung“ oder „Soll-Quote“ bezeichnet. Solche unverbindlichen Regelungen gab es in der Vergangenheit in mehreren österreichischen Gewerkschaften. Sie erwiesen sich (so wie auch in Deutschland) als wenig wirkungsvoll und führten nicht zur proportionalen Repräsentation von Frauen (Blaschke, 2008: 177, 244; vgl. auch Plogstedt, 2015: 290, 313).

Gewerkschaftliche Frauenquoten legen einen bestimmten (Mindest-)Frauenanteil in einem Gremium oder einer Position (z.B. Abteilungsleitung) fest. Sie können entweder einen spezifischen mindestens zu erreichenden Frauenanteil vorschreiben (wie z.B. in der Schweizer Gewerkschaft Unia - Monney, Fillieule, \& Avanza, 2013: 36) oder die Höhe des zu erreichenden Frauenanteils vom Frauenanteil an den Mitgliedern abhängig machen (proportionale Repräsentation). Im zweiten, gängigeren Fall kann für Gremien bzw. Positionen von Subeinheiten (z.B. regionale oder fachliche Untergliederungen) entweder der Frauenanteil an den Mitgliedern insgesamt herangezogen werden oder der Frauenanteil an den Mitgliedern der jeweiligen Subeinheit. Frauenquoten können für alle Organe bzw. Positionen gelten oder nur für bestimmte. Üblicherweise beziehen gewerkschaftliche Frauenquoten sich auf Organe bzw. Gremien, welche im Wesentlichen aus gewählten FunktionärInnen bestehen; es ist aber möglich, dass eine Frauenquote auch für die angestellten, hauptamtlichen SekretärInnen gilt (wie z.B. in der deutschen ver.di - Kirsch \& Blaschke, 2014: 208).

Während in Deutschland im Laufe der 1990er Jahren mehrere Gewerkschaften eine Quotenregelung zur proportionalen Vertretung von Frauen in den Entscheidungsstrukturen einführten, machte dies in Österreich damals nur die Gewerkschaft der Privatangestellten (GPA) (1997). Erst nachdem der Dachverband, der Österreichische Gewerkschaftsbund (ÖGB), die Einführung einer Quotenregelung für seine Organe (welche von den Mitglieds-

2 In westlichen Staaten dominieren freiwillige Quotenregelungen der politischen Parteien, in anderen Regionen sind gesetzliche Quotenregelungen häufiger (Dahlerup 2006b: 293-294). 
gewerkschaften beschickt werden) beschlossen hatte, haben mehrere österreichische Gewerkschaften eine solche für ihre Gremien eingeführt. Die österreichischen Gewerkschaften sind formal nicht eigenständig, sondern Untergliederungen des ÖGB, und ihre Geschäftsordnungen orientieren sich an den Statuten und der Geschäftsordnung des ÖGB. Dies hat die Aufnahme der Frauenquote in die Geschäftsordnungen der Einzelgewerkschaften begünstigt, jedoch bisher nicht zwingend dazu geführt: Die Gewerkschaft Öffentlicher Dienst hat die Frauenquote bislang nicht übernommen (ein entsprechender Antrag der Frauenabteilung wurde am Bundeskongress 2011 de facto abgelehnt - Sorger, 2014: 239). Und nicht in jeder Gewerkschaft gilt die Frauenquote für alle Organe, sondern bloß für einige (z.B. Gewerkschaft Bau-Holz, 2010). Die Frauenquoten der österreichischen Gewerkschaften beziehen sich nur auf die gewählten Gremien, der zu erreichende Frauenanteil entspricht dem Frauenanteil an den Mitgliedern, bei Subbereichen dem Frauenanteil an den Mitgliedern der jeweiligen Organisationseinheit.

In Gewerkschaften haben - so wie in politischen Parteien (Krook, 2008: 347) - Quotenregelungen ihren Ursprung fast immer in den Forderungen von Frauen innerhalb und außerhalb der Organisation, die eine bessere Repräsentation verlangen. Was sind nun die Gründe dafür, dass die Männer und diejenigen Frauen, die nicht zu den Proponentinnen gehören, diesen Forderungen zustimmen? Für den politischen Bereich (Parteien, Parlamente) existieren mehrere Untersuchungen; zusammenfassende Übersichten nennen mehrere Motive für die Einführung von Quotenregelungen, darunter die Befürwortung von Gleichberechtigung, wahltaktische Überlegungen (v.a. wenn politische Gegner eine Quote eingeführt haben) und eine Erhöhung der Legitimität bzw. die Absicht, sich (auch aus wahltaktischen Gründen) ein modernes, progressives Image zu geben (Dahlerup, 2006b: 295; Krook, 2008: 352-354). Zu den Motiven in Gewerkschaften gibt es bisher keine systematisierende Darstellung, jedoch einige empirische Befunde. Bei der Gründung der Gewerkschaft ver.di werden folgende Gründe für die Befürwortung der Frauenquote durch die Männer identifiziert: Die Frauenstrukturen der Gründungsgewerkschaften beabsichtigten, die Quote zur Bedingung ihrer Zustimmung zur Fusion zu machen; das Ziel der Gleichstellung der Geschlechter war unumstritten; die Öffentlichkeit erwartete eine Quote; die Gewerkschaft erhoffte sich mehr weibliche Mitglieder; und das Fehlen der Quote wäre für die Frauen derjenigen Gründungsgewerkschaften, die bereits eine Quotenregelung gehabt hatten, ein Rückschritt gewesen (Kirsch \& Blaschke, 2008: 205-206). Für den Quotenbeschluss einer Vorgängergewerkschaft von ver.di (ÖTV) legt eine Untersuchung nahe, dass die männliche Zustimmung zum Antrag der Frauenstruktur wesentlich von der Einschätzung der positiven Außenwirkung getragen war (Kassel, 2001: 99). Die Einführung einer (de-facto-)Quotenregelung bei der Gründung der britischen Gewerkschaft UNISON erfolgte aufgrund des Drucks, den die Frauen ausübten, und aufgrund der Notwendigkeit, angesichts politischer Veränderungen die gewerkschaftliche Organisation fundamental zu ändern und insbesondere die innergewerkschaftliche Demokratie zu verbessern (Terry, 1996).

Im Anschluss an diese Befunde können folgende mögliche Motive für die Einführung von Frauenquoten in Gewerkschaften angeführt werden, welche gleichzeitig auftreten können: die prinzipielle Haltung, dass Frauen gleichberechtigt integriert zu sein haben; interne Frauengruppen, die die Frauenquote fordern und über wirksame Druckmittel verfügen; die Absicht, Mitglieder zu mobilisieren; das Streben nach einem progressiven, modernen 
Image, um darüber mehr Mitglieder zu gewinnen; eine Erhöhung des Einflusses innerhalb der Gewerkschaftsbewegung sowie in der Gesellschaft mittels eines verbesserten öffentlichen Images; und (primär ebenfalls die Außenwirkung betreffend) ein Druck zur Einführung von Quotenregelungen, wenn andere Gewerkschaften solche bereits eingeführt haben.

Gewerkschaftliche Frauenquoten sollen zur proportionalen Repräsentation von Frauen in den betreffenden Gremien und Positionen führen. Damit verbunden ist die Erwartung, dass Frauen als Gruppe ein stärkeres Gewicht in der Gewerkschaft bekommen und ihre Anliegen in der Gewerkschaftspolitik mehr Beachtung finden. Wie Healy \& Kirton (2000: 357-358) und Cockburn (1991: 115-119, 215-236) ausführen, muss dies jedoch nicht der Fall sein, die männlichen Oligarchien und die männlich geprägte Organisationskultur können trotz Quotenregelung weiterhin bestehen bleiben. Die Mechanismen, die zuvor Frauen benachteiligt und zur Unterrepräsentation von Frauen geführt haben, verschwinden nicht automatisch durch die Quote und können sogar die Erfüllung derselben behindern. In Arbeiten zu politischen Parteien und Quotenregelungen in der Wirtschaft wird deshalb der Schluss gezogen, dass für eine erfolgreiche Umsetzung häufig sanktionsbewehrte Regelungen in Verbindung mit Berichtspflichten und anderen Begleitmaßnahmen (z.B. Förderung der Familienfreundlichkeit) notwendig sind (Bothfeld \& Rouault, 2015; Matland, 2006). ${ }^{3}$

Die Existenz einer Frauenquote bedeutet demnach nicht zwingend, dass Fraueninteressen in der Gewerkschaft mehr Beachtung finden. Allerdings kommen mehrere Autorinnen zu dem Schluss, dass die Beteiligung von Frauen (die durch eine Frauenquote in einem bestimmten Ausmaß gesichert ist) eine zwar nicht hinreichende, aber notwendige Bedingung dafür ist, dass Frauenanliegen in der Gewerkschaftspolitik berücksichtigt werden (z.B. Colgan \& Ledwith, 2002: 172, 184-185; Dickens, 2000: 203-205; McBride, 2001: 142, 180). Eine Untersuchung zum Effekt von Frauenquoten in deutschen und österreichischen Gewerkschaften zieht das Fazit, dass die aufgrund der Frauenquote gestiegene Präsenz von Frauen zur vermehrten Berücksichtigung von Frauenanliegen beigetragen hat (Kirsch \& Blaschke, 2014: 215).

\section{Forschungsdesign}

Die Fallauswahl war geleitet von Ergebnissen einer Studie Anfang des Jahrtausends, wonach zum einen in Österreich und Deutschland in den ArbeiterInnengewerkschaften des privaten Dienstleistungssektors Frauen in Gremien und Positionen deutlich unterrepräsentiert sind und zum anderen eine höhere Qualifikation der Mitglieder bei der Besetzung der Spitzenpositionen durch Frauen einen positiven Effekt zeigt (Blaschke, 2008, 2015). Weiters ergaben sich auf der Ebene der Kollektivverträge Hinweise darauf, dass eine höhere Qualifikation und der Angestelltenstatus der betroffenen ArbeitnehmerInnen bzw. Mitglieder Lohnabschlüsse begünstigen, die zum Schließen der geschlechtsspezifischen Einkommensdifferenzen beitragen (Blaschke, 2011). Im Anschluss an diese Befunde sollten eine ArbeiterInnengewerkschaft des privaten Dienstleistungssektors mit (im Durchschnitt) nied-

3 Auch in Bezug auf betriebliche Gleichstellungsmaßnahmen generell stellen Bothfeld, Hübers und Rouault fest, dass es sanktionsbewehrter rechtlicher Vorschriften und verbindlicher prozeduraler Regelungen (incl. eines Monitoring-Systems) bedarf (2010) 
riger qualifizierten Mitgliedern und eine Gewerkschaft, deren Mitglieder aus Angestellten sowie (im Durchschnitt) höher Qualifizierten besteht, miteinander verglichen werden. Die Untersuchung richtet sich auf die Prozesse, die am Beispiel der Frauenquote zur Ausgestaltung der Teilhabe von Frauen und der Vertretung ihrer Interessen beitragen.

Als Gewerkschaft, deren Mitglieder Angestellte und höher Qualifizierte sind, wird die GPA-djp untersucht, sie hatte zum Zeitpunkt der Erhebung 45\% weibliche Mitglieder. Das Qualifikationsniveau der Mitglieder der GPA-djp liegt über dem Durchschnitt, zwischen den einzelnen Wirtschaftsbereichen (der Branchenstruktur der GPA-djp) gibt es allerdings Unterschiede. Bei der Auswahl von InterviewpartnerInnen auf der Ebene der Wirtschaftsbereiche erfolgte eine stärkere Berücksichtigung von Bereichen mit höherer Qualifikation (z.B. Banken - siehe unten).

Als Gewerkschaft, deren Mitglieder ArbeiterInnen und niedriger Qualifizierte des privaten Dienstleistungssektors sind, wird die Gewerkschaft vida untersucht, sie hatte zum Zeitpunkt der Erhebung 32\% weibliche Mitglieder. Die Gewerkschaft vida ist im Jahr 2006 aus der Fusion von zwei ArbeiterInnengewerkschaften des privaten Dienstleistungsbereichs - Gewerkschaft Handel, Transport, Verkehr (HTV) und Gewerkschaft Hotel, Gastgewerbe, persönliche Dienste (HGPD) - mit der Gewerkschaft der Eisenbahner (GdE) hervorgegangen. (Die GdE organisierte alle ArbeitnehmerInnen ihrer Branche, welche früher zum öffentlichen Sektor gehörte.) Die Gewerkschaft vida war zum Zeitpunkt der Erhebung in drei Sektionen gegliedert. Die Auswahl von InterviewpartnerInnen auf der Sektionsebene war innerhalb der beiden Sektionen geplant, in denen die Branchen der HTV und HGPD größtenteils vertreten waren (siehe unten).

Das wesentliche Erhebungsinstrument, neben der Analyse von Geschäftsordnungen und Internetseiten, waren leitfadengestützte Interviews. Hauptthemen der Interviews waren die Einführung und die Umsetzung der Frauenquote in den betreffenden Gewerkschaften. ${ }^{4}$ In Bezug darauf wurden die GesprächspartnerInnen als ExpertInnen befragt, die „Auskunft über ihr eigenes Handlungsfeld geben“ (Meuser \& Nagel 1991: 445). Das wesentliche Anliegen der Analyse ist die Rekonstruktion der betreffenden Prozessabläufe, dazu zielt sie vor allem auf das Prozesswissen der Befragten ab (vgl. Bogner, Littig, \& Menz, 2014: 1822). Daneben ist das Deutungswissen ebenfalls relevant, vor allem in der Form von Interpretationen des Verhaltens anderer. Durch das Interviewen mehrerer Personen in teilweise unterschiedlichen Positionen und eine Zusammenschau ihrer Perspektiven, die sich ergänzen oder auch kontrastieren, soll eine möglichst umfassende Rekonstruktion erreicht werden. Neben den Handlungen in Zusammenhang mit der Einführung und Umsetzung der Frauenquote sollen auch die hinter den Handlungen liegenden Motive, Absichten und Einstellungen sowie die strukturellen Bedingungen der Handlungen betrachtet werden. Die Erfassung erfolgt zum einen über Interviewaussagen der Befragten zur eigenen Handlungspraxis etc. und zum anderen über die Aussagen der Befragten zu den Handlungen etc. anderer relevanter GewerkschafterInnen.

4 Weitere Themen der Interviews waren: die Einführung und Umsetzung der Frauenquote im ÖGB aus der Perspektive der jeweiligen Gewerkschaft, die Präsenz von Frauenanliegen in den Kollektivvertragsverhandlungen, die subjektive Bewertung der gewerkschaftlichen Tätigkeit (was gefällt der befragten Person, was würde sie ändern) und (bei weiblichen Befragten) förderliche sowie hemmende Bedingungen des persönlichen gewerkschaftlichen Engagements. 


\section{Forschungsleitende Annahmen}

Hintergrund der Arbeit ist ein Rahmen, der mehrere theoretische Perspektiven der Organisationssoziologie und ihre Annahmen integriert. ${ }^{5}$ Zum einen ist dies die Annahme des Einflusses der Umwelt auf die Organisation, die aus der, der Kontingenztheorie ähnlichen, soziotechnischen Integrationstheorie kommt (Thompson, 1967). Eine mögliche Form des Einflusses der Umwelt ist die Übernahme von Institutionen durch die Organisation zwecks Sicherung ihrer Legitimität, wie sie der Neo-Institutionalismus darlegt (z.B. Meyer \& Rowan, 1977). Eine andere Form des Einflusses der Umwelt auf die Organisation (insbesondere hinsichtlich sozialer Normen, Verhaltensmuster und Werteinstellungen) erfolgt über die aus der Umwelt kommenden Mitglieder (z.B. Scott, 1986: 41). Dazu, wie die Entscheidungen zur Ausgestaltung der Organisation zustande kommen, wird auf die verhaltenswissenschaftliche Entscheidungstheorie und den mikropolitischen Ansatz zurückgegriffen (z.B. Berger \& Mehlich, 1999; Riegraf, 1996: 77-110): Die AkteurInnen orientieren sich in ihrem Verhalten nicht nur an den Normen und Zielen der Organisation, sondern auch an ihren individuellen Interessen und Präferenzen; organisationale Entscheidungen sind das Ergebnis von Aushandlungsprozessen, auf deren Ergebnis die Machtverteilung der beteiligten AkteurInnen einen wesentlichen Einfluss nimmt. Sowohl Merkmale der Organisation als auch der Umwelt spielen auf der Akteursebene eine Rolle, sie beeinflussen u.a. individuelle Interessen, Situationsdefinitionen und wahrgenommene Handlungsalternativen. Über den Einfluss der Umwelt sowie der Merkmale der Organisation wird die strukturtheoretische Perspektive der Erklärung der Geschlechterungleichheit berücksichtigt, mit dem Blick auf die Entscheidungsprozesse die handlungstheoretische.

Diese theoretischen Annahmen, Hinweise aus einer früheren empirischen Erhebung (Blaschke, 2008, 2015), die oben dargestellten Motive für die Einführung einer Quotenregelung (mit Erweiterung auf Geschlechtergleichstellung generell), Ergebnisse aus Studien zur politischen Partizipation und Plausibilitätsüberlegungen erlauben die Formulierung von Annahmen zu den Prozessen, die zu Differenzen in der Teilhabe von Frauen zwischen den beiden untersuchten Arten von Gewerkschaften führen können.

Auf Seite der Frauen können sich, neben der Qualifikation, Unterschiede in den folgenden Bereichen, die Bedingungen ihres Handelns darstellen, auswirken: Arbeitsbedingungen, Verhältnisse in der Gewerkschaft und Belastung durch private Versorgungsarbeit. Auf diese Bereiche übt die Qualifikation einen Einfluss aus oder könnte einen solchen ausüben. Berufsstatus und Qualifikationsniveau bestimmen in hohem Ausmaß die Arbeitssituation; Arbeitsbelastung und andere Arbeitsbedingungen wiederum beeinflussen die Möglichkeiten und die Bereitschaft der Frauen zum Engagement in gewerkschaftlicher Frauenpolitik. Mehrere der in niedrig qualifizierten Tätigkeiten, insbesondere im privaten Dienstleistungsbereich, tendenziell häufiger auftretenden Merkmale der Arbeitssituation wirken sich hinderlich aus: Körperlich schwere und belastende Arbeit reduziert die Bereitschaft zu ehrenamtlicher Tätigkeit; unregelmäßige Arbeitszeiten an Abenden und Wochenenden (teilweise verbunden mit Teilzeitarbeit) erschweren das individuelle Engagement und auch kollektives Handeln, da keine gemeinsamen Termine für gewerkschaftliche Tref- 
fen gefunden werden. Höher qualifizierte Tätigkeiten weisen in dieser Hinsicht tendenziell bessere Voraussetzungen auf. Höhere Bildung kann sich auch noch auf andere Weise positiv auf die Möglichkeiten und die Bereitschaft zum individuellen Engagement auswirken. Einerseits kann vermutet werden, dass mit höherer Bildung und höherem Einkommen die Frauen sich mehr von der Hausarbeit entlasten können, wodurch Zeit für ehrenamtliche Tätigkeit frei wird. Andererseits kann davon ausgegangen werden, dass Frauen mit höherer Bildung mehr Selbstsicherheit besitzen, sich besser in ihre Gewerkschaft einbringen können und auch mehr Interesse an aktivem Engagement haben (vgl. Hoecker, 1995: 33-34). Generell führt höhere Bildung zu höherer politischer Partizipation (z.B. Lippert, 2002: 148, 322324; Sebinger \& Nemella, 2005: 456-459). Schließlich kann die Beteiligung von Frauen durch die Gewerkschaftsstruktur und -kultur beeinflusst werden, welche in Abhängigkeit vom Qualifikationsniveau der Mitglieder unterschiedlich ausgestaltet sein können.

Im Hinblick auf das Verhalten männlicher Gewerkschafter können weniger leicht eindeutige Annahmen zu Unterschieden zwischen geringer und höher qualifizierten Männern aufgestellt werden. Zwar steigt auf der Einstellungsebene die Befürwortung egalitärer Geschlechterarrangements mit höherer Bildung, sei es aus persönlicher Überzeugung oder unter normativem Druck (z.B. Schulz \& Hummer, 2005: 354-356). Aber wie u.a. Wetterer (2006) anhand mehrerer Berufsfelder darlegt, finden diskursiv geäußerte Gleichberechtigungsnormen des Öfteren keinen Niederschlag in den Interaktionen, sondern letztere reproduzieren die tradierten Geschlechterungleichheiten immer wieder aufs Neue. Für den Bereich der privaten Versorgungsarbeit zeigt sich, dass die verbal kundgetane egalitäre Einstellung hochgebildeter Männer sich nicht in einem egalitären Verhalten niederschlägt (Koppetsch \& Burkart, 1999: 215-220). Die Gewerkschaften stellen ein spezifisches Handlungsfeld dar, das in dieser Hinsicht bisher nicht untersucht wurde. Möglich wäre, dass besser gebildete Männer hier ein egalitäreres Verhalten zeigen als weniger qualifizierte Männer, weil sie in diesem öffentlichen Bereich einen stärkeren normativen Druck in Bezug auf ihr Verhalten verspüren (vgl. Behnke \& Meuser, 1999: 68), oder weil sie darin eher einen Nutzen für ihre Gewerkschaft sehen.

\section{Ablauf der Untersuchung}

Für eine möglichst umfassende Rekonstruktion der zu untersuchenden Prozesse sollten die Wahrnehmungen von Personen in unterschiedlichen Bereichen und Positionen erfasst werden, weshalb Angehörige verschiedener Personengruppen (in Bezug auf Geschlecht, Haupt-/ Ehrenamtlichkeit, Branchen, u.a.) befragt wurden. Insgesamt wurden im Zeitraum November 2013 bis Juli 2014 durch das Forschungsteam 41 Interviews mit einer durchschnittlichen Dauer von 55 Minuten durchgeführt: 17 in der Gewerkschaft vida, 24 in der GPA-djp. In der vida konnten (wie geplant) ungefähr gleich viele Frauen (9) wie Männer (8) für ein Gespräch gewonnen werden. In der GPA-djp ergab sich ein starker Überhang an weiblichen Befragten: 16 Frauen gegenüber 8 Männern. Das Verhältnis von hauptamtlichen zu ehrenamtlichen GewerkschafterInnen betrug 16 zu 25, mit annähernd gleichem Verhältnis in vida und GPA-djp.

Bei der Branchenauswahl erfolgte eine Konzentration auf bestimmte Branchen. In der GPA-djp wurde aus allen größeren Wirtschaftsbereichen - ausgenommen denjenigen mit 
einem sehr niedrigen Frauenanteil - zumindest eine Vertreterin bzw. ein Vertreter interviewt, wobei entsprechend der Forschungsfragestellung Branchen mit höherem Qualifikationsniveau etwas stärker vertreten sind. Die GesprächspartnerInnen der GPA-djp kamen aus folgenden Wirtschaftsbereichen: Elektro/IT/Telekom, Finance, Forschung/Bildung/Kultur/Organisationen, Handel, Medien/JournalistInnen und Metall/Bergwerke. In der vida wurde auf die Sektionen „Soziale, Persönliche Dienste und Gesundheitsberufe“ und „Private Dienstleistungen“ fokussiert, es wurde mit Personen aus folgenden Branchen gesprochen: Gebäudemanagement, Gesundheit, Soziale Dienste, Tourismus und (ungeplanterweise) Eisenbahn (eine Person). Auffällig war, dass es in der vida deutlich schwieriger war, InterviewpartnerInnen zu gewinnen, insbesondere im ehrenamtlichen Bereich.

Beim spezifischen Thema der Gespräche antworten Interviewte, insbesondere männliche Interviewte, gegenüber einer interviewenden Person desselben Geschlechts möglicherweise ehrlicher als gegenüber einer Person des anderen Geschlechts. ${ }^{6}$ Deshalb wurden die Interviews mit männlichen Befragten überwiegend vom männlichen Mitarbeiter durchgeführt, die Interviewtätigkeit der weiblichen Mitarbeiterin konzentrierte sich stärker auf die weiblichen Befragten.

Die Gespräche wurden aufgezeichnet und größtenteils wörtlich transkribiert. Das Vorgehen bei der Auswertung des Materials fällt unter den Typ einer inhaltlich strukturierenden qualitativen Inhaltsanalyse (Kuckartz, 2012: 77-84). Die Grobkategorien der Auswertung wurden von den Forschungsfragen abgeleitet. Die unterste Ebene der Subkategorien wurde aus dem Material generiert (induktive Kategorienbildung). Die Kategorien auf den Ebenen zwischen Grobkategorien und untersten Subkategorien wurden mittels einer Kombination aus deduktiver und induktiver Kategorienbildung gewonnen (vgl. Mayring, 2003; Schreier, 2012).

\section{Ergebnisse zur Einführung der Frauenquote}

\subsection{Ablauf}

In den 1990er Jahren wurde die Frauenquote in der GPA (die damals noch nicht mit der Gewerkschaft Druck, Journalismus, Papier fusioniert war) im Zuge eines Organisationsentwicklungsprozesses als eine von mehreren Reformmaßnahmen eingeführt. Die wesentliche Initiatorin für die Einführung war eine zu dieser Zeit tätige Frauensekretärin, die dann auch im Prozess der Einführung eine zentrale Rolle einnahm. Nach einer intensiven Diskussion in der Frauenabteilung und Erarbeitung einer Quotenregelung beschloss der Frauenzentralausschuss 1996, dass diese Regelung in der GPA eingeführt werden solle (ÖGBFrauenabteilung, 1997: 28). Für ein Inkrafttreten war allerdings noch ein Beschluss der Gesamtorganisation notwendig.

Ein zweiter wichtiger Akteur neben der Frauensekretärin war der damalige Zentralsekretär, der die Quotenregelung mitentwickelte und die Frauensekretärin in das Kernteam des Organisationsentwicklungsprozesses einband. Die Organisationsreform in der GPA wird

6 Zur Rolle des Geschlechts in der Interaktion Interview vgl. Abels \& Behrens (2002), Diekmann (2012: 466) und Littig (2002). 
von mehreren Befragten als günstige Gelegenheit beschrieben, die Quotenregelung einzuführen.

Gw6: Und was [...] sich sehr gut getroffen hat, war, dass [der damalige, erst seit kurzem im Amt befindliche Zentralsekretär] angeleiert hat einen Prozess der Organisationsentwicklung in der GPA. Und das war natürlich eine gute Energie für eine Quotendebatte. Weil es war immer klar, wenn du einmal die Organisation komplett reflektierst, evaluierst, was gut läuft, was weniger gut läuft, was auch sozusagen machtpolitisch wesentlich ist und dann daraus ein neues Statut schreibst, dann ist das jetzt der Zeitpunkt Frauenquoten zu verankern. (GPA-djp, weiblich, hauptamtlich, Bundesebene, leitende Funktion)

Ebenso wie in der GPA wurde in der vida die Frauenquote im Zuge einer organisatorischen Änderung eingeführt, welche allerdings grundsätzlicherer Art war, denn es handelte sich um eine Neugründung aufgrund einer Fusion im Jahr 2006. In keiner der drei Gründungsgewerkschaften der vida gab es eine Quotenregelung und nur in einer Gewerkschaft (der HGPD) eine sogenannte „Soll-Regelung“ für bestimmte Gremien (HGPD, 1995). Im Prozess der Fusion der drei Gewerkschaften wurde die Frauenquote von den Frauen der Frauenabteilungen der drei Gewerkschaften eingebracht. So wie in der GPA kam die Initiative von den in der Frauenabteilung aktiven Gewerkschafterinnen. Zur gleichen Zeit lief auch die Diskussion zur Einführung der Frauenquote im Dachverband ÖGB, was ein günstiges Klima für die Einführung einer Quotenregelung in der durch Fusion neu zu gründenden Gewerkschaft schuf.

Vw3: [...] von den Frauen selber ist [die Frauenquote] eingebracht worden und es war nicht so überraschend, weil sie in der Gesamtorganisation, in den einzelnen Fachgewerkschaften, überall war es ein Thema und ein Punkt. Und daher war es weder eine Überraschung noch eher unüblich, also es war irgendwie eine klare Sache, das gehört dazu. (vida, weiblich, hauptamtlich)

Die Organisationsreform der GPA war insgesamt von intensiven, breit angelegten Diskussionen in der Gewerkschaft begleitet und auch zur Vorbereitung des Beschlusses der Quotenregelung wurden ca. zwei Jahre lang Diskussionen geführt. Dass die Frauenquote in der (damaligen) GPA nach einer längeren, weite Teile der FunktionärInnen miteinbeziehenden Diskussion eingeführt wurde, wird von einer Befragten explizit als Spezifikum der GPA-djp als Angestelltengewerkschaft gesehen (im Unterschied zu den ArbeiterInnengewerkschaften). In der vida hingegen fanden Diskussionen dazu, ob die Frauenquote eingeführt werden soll, offensichtlich nur in kleineren Zirkeln statt. Denn die Angaben dazu, ob es überhaupt Diskussionen gab, differieren und einige Interviewte berichten von Gesprächen, an denen sie selbst nicht beteiligt waren. Dass die Diskussionen im Vorfeld nicht breit geführt wurden, zeigt sich auch darin, dass eine Interviewte erwähnt, dass sie vom Quotenbeschluss erst im Nachhinein erfuhr. Die nicht direkt an den Diskussionen Beteiligten unter den GesprächspartnerInnen der vida können häufig nur vage Angaben zum Ablauf der Einführung machen. Mehrere ziehen einen direkten Zusammenhang zum Beschluss der Frauenquote im ÖGB.

Vw4: [Die Frauenquote] ist vom ÖGB vorgeschrieben worden und sie haben sich nachher nicht, mehr oder minder, davor drücken können. (vida, weiblich, ehrenamtlich, Regionalebene)

Der Beschluss der Frauenquote in der vida fand Ende 2006 statt, wenige Wochen vor dem Beschluss der Frauenquote im ÖGB. Letztere wurde am Bundeskongress im Januar 2007 verabschiedet; es war jedoch schon mehrere Monate vorher festgelegt worden, dass die Frauenquote im ÖGB kommen werde. Auffällig ist, dass die Erzählungen der Befragten 
zum Ablauf der Einführung der Quotenregelung sich in der GPA-djp sehr stark decken und kaum Abweichungen auftreten, während in der vida die Angaben der Befragten zum Prozess der Einführung teilweise differieren. Letzteres weist darauf hin, dass die Einführung in der vida auf eine Weise ablief, die in unterschiedlichen Positionen zu unterschiedlichen Wahrnehmungen führte, und dass ein Teil der Befragten aus der vida nicht nur nicht eingebunden war, sondern auch keine ausreichenden bzw. übereinstimmenden Informationen über den Ablauf und die Hintergründe erhielt.

In beiden Gewerkschaften gab es GegnerInnen und SkeptikerInnen der Quote, vor allem unter den Männern. Dies wird in der GPA-djp von allen Befragten, die sich zum Prozess der Einführung äußern, angeführt. In der vida, in der es weniger Diskussionen gab, berichtet nur ein Drittel der Befragten von GegnerInnen bzw. SkeptikerInnen. Die in GPA und vida genannten von Männern vorgebrachten Argumente gegen die Einführung der Frauenquote decken sich zu einem großen Teil. Aus Platzgründen kann hier auf sie nicht näher eingegangen werden.

In der GPA wurde die Quotenregelung nicht nur von der Frauenabteilung und von Frauen auf verschiedenen Ebenen der Organisation befürwortet, sondern laut mehreren Befragten auch von Männern einschließlich solcher in Führungspositionen. In der vida wird dies seltener angegeben und bezieht sich primär auf Männer in Spitzenpositionen. Als Motive dafür, warum Männer für die Einführung der Frauenquote waren, werden von den Befragten der GPA-djp (von denen einige selbst männliche Befürworter der Quote waren) und von den Befragten der vida größtenteils solche genannt, die auf den Nutzen für die Gewerkschaft abzielen. Am häufigsten wird angeführt, dass eine Frauenquote bzw. die dadurch erreichbare Präsenz von Frauen in den Gremien und Positionen notwendig für eine erfolgreiche Organisation sind. Dass Frauen eine Bereicherung für die Organisation sind, wird mehrmals angegeben. Das öfters genannte Argument, dass Frauen eine wichtige Mitgliedergruppe sind und deshalb adäquat repräsentiert zu sein haben, kann sowohl das Bemühen um Herstellung von Geschlechtergerechtigkeit ausdrücken als auch den Nutzen für die Organisation (damit weibliche Mitglieder sich nicht aufgrund mangelnder Repräsentation zurückziehen bzw. potentielle weibliche Mitglieder angezogen werden). Aus der Aussage eines männlichen Spitzenfunktionärs der vida (der keine persönlichen Angaben machte) lässt sich schließen, dass die Frauenquote der ÖGB-Statuten für die Entscheidungsträger ein wichtiger Grund für die Einführung in der eigenen Gewerkschaft war.

\subsection{Beschluss der Quote}

In der GPA wurde die Quotenregelung (Frauenquote für alle Gremien) 1997 im Zentralvorstand beschlossen und beim Gewerkschaftstag 1998 als Beschluss der Gesamtorganisation bestätigt sowie in die Wahl- und Geschäftsordnung aufgenommen (ÖGB-Frauenabteilung, 1998: 31, 1999: 41). Bis 2002 waren als Übergangsregelung Ausnahmen vorgesehen, wenn in einem bestimmten Bereich die Quote der gewählten Betriebsrätinnen mehr als $15 \%$ unter dem Frauenanteil bei den Mitgliedern liegt. Weiters war eine Aufstockung von Gremien möglich, um die Quote zu erreichen (GPA, 1998, 2000). Beim Gewerkschaftstag 2000 wurde Letzteres modifiziert: Gremien müssen seitdem um Frauen erweitert werden, wenn die Frauenquote nicht erfüllt ist, diese zusätzlichen Frauen werden durch das jeweilige Frauengremium gewählt (GPA, 2000). 
Die Frauenquote der vida wurde beim Gründungsgewerkschaftstag Anfang Dezember 2006 beschlossen und in die Statuten aufgenommen. Sie gilt (laut den zum Erhebungszeitpunkt weiterhin aktuellen Statuten aus dem Jahr 2006) für die Delegierten zum Gewerkschaftstag, für den Bundesvorstand, für die Bundessektionsvorstände und -präsidien, für die Bundesfachgruppenvorstände und für die Landespräsidien sowie Landesvorstände. Falls zu wenige Frauen gewählt werden, kann für die meisten dieser Gremien (ausgenommen sind der Gewerkschaftstag und die Landesvorstände) die Frauenabteilung zusätzliche Vertreterinnen entsenden, bis der notwendige Frauenanteil im Gremium erreicht ist (vida, 2006). Die Frauenquote gilt somit nicht für alle Organe (u.a. sind das Präsidium und die Landeskonferenzen ausgenommen).

Sowohl in der GPA als auch der vida wird die Befürwortung durch die Gewerkschaftsspitze (im Fall der GPA durch den Vorsitzenden und die BundesgeschäftsführerInnen, im Fall der vida durch die Vorsitzenden der drei Gründungsgewerkschaften) als wichtig für deren Beschluss gesehen.

Gw8: [...], den [Vorsitzenden] mussten wir natürlich auch überzeugen, und wenn der bei dem Bundesvorstand seinerzeit, wo das zur Beschlusslage vorlag, nicht von oben gesagt hätte „Und ich bin für diese Frauenquote. Und ich glaube, dass es höchst an der Zeit ist“" und so, dann wäre es sicherlich nicht so positiv ausgegangen. Die Männer haben sich natürlich orientiert, erstens an dem Vorsitzenden und zweitens auch an einem Mann. (GPA-djp, weiblich, ehrenamtlich, Bundesebene, leitende Funktion)

Vw6: Die drei ehemaligen Vorsitzenden haben uns von Anfang an unterstützt und das war natürlich sehr hilfreich, ja, wenn das so top-down passiert ((2)) gibt es kaum Widerstand, sagen wir einmal so. Aus welchen Gründen auch immer ((lacht)) (vida, weiblich, hauptamtlich)

Der Beschluss der Frauenquote im Vorstand und darauffolgend am Gewerkschaftstag erfolgte in der GPA dann zwar nicht einstimmig, aber mit einer sehr großen Mehrheit. In der vida waren die Diskussionen pro und contra Frauenquote innerhalb der Fraktionen geführt worden (die österreichischen Gewerkschaften sind zwar Einheitsgewerkschaften, intern existieren aber politische Fraktionen); nachdem dort der Beschluss gefasst worden war (evtl. nicht einstimmig - S.B./M.M.), war der Beschluss am Gewerkschaftstag einstimmig.

\section{Ergebnisse zur Umsetzung der Frauenquote}

\subsection{Erfüllung der Quote}

Die aktuelle Situation in Bezug auf die Erfüllung der Frauenquote wird von den Befragten entlang unterschiedlicher Dimensionen beschrieben. Öfter formuliert wurde „funktioniert gut - weniger gut“ „Quote wird eingehalten - nicht eingehalten“ „funktioniert gut - ist schwierig“ „Nachnominierungen nicht notwendig - notwendig“. Eine wechselseitige Überführung der Angaben zu den Dimensionen ist mit Unschärfen behaftet, dies ist bei der Interpretation der Befunde zu berücksichtigen. Ebenso ist zu beachten, dass mehrere Befragte in einem Teilbereich (Wirtschaftsbereich, Region) tätig sind und ihre Aussagen sich (auch ohne dass sie dies explizit sagen) primär auf diesen Bereich beziehen.

In der GPA-djp gibt die Mehrheit der Befragten an, dass die Umsetzung der Frauenquote gut läuft, wenn auch in manchen Bereichen Nachnominierungen notwendig sind oder 
Schwierigkeiten auftreten (u.a. nach dem Ausscheiden einer Frau). Einige betonen, dass (teilweise) Kontrolle notwendig ist. Lediglich zwei Befragte geben an, dass in bestimmten Gremien die Frauenquote nicht eingehalten wird bzw. werden kann. Letzteres widerspricht anderen Aussagen, dass die Quote (wenn auch mit Nachnominierungen) ,immer“ eingehalten werde, es handelt sich jedoch, nach den Angaben in den Gesprächen, um einige wenige Ausnahmen in untergeordneten Bereichen.

In den ersten Jahren nach Einführung der Frauenquote in der GPA wurde von der Übergangsregelung und von der Aufstockungsmöglichkeit häufiger Gebrauch gemacht. Die Kontrolle der Einhaltung hatte damals noch Schwächen und es kam auch vor, dass zur Erfüllung der Quote Frauen nominiert wurden, die sich nicht engagierten. Ein paar Jahre nach ihrer Einführung (zum Zeitpunkt der nächsten Wahlen im Jahr 2002) hatte sich die Frauenquote in der GPA dann eingespielt, nur eine Befragte meint, dass es sehr lange gedauert hätte, bis die Frauenquote ,wirklich greift" (Gw16).

Im Gegensatz zur GPA-djp bezeichnen nur wenige Befragte der vida die aktuelle Situation der Umsetzung der Frauenquote als ,,(relativ) gut“ (Vm3, Vm5) oder geben an, dass sie eingehalten wird. Diese positiven Aussagen stehen im Widerspruch zu anderen, wonach die Frauenquote des Öfteren nicht eingehalten wird, und beziehen sich evtl. nur auf eine Region bzw. nur auf eine Sektion, obwohl sie allgemein formuliert sind. Mehr als ein Drittel der Befragten gibt an, dass die Frauenquote in manchen bzw. bestimmten Bereichen nicht eingehalten wird.

Vw5: Und nein, sie haben die Frauen nicht drinnen, sie haben / sie erfüllen absolut die Quote nicht. (vida, weiblich, ehrenamtlich, Regional- und Bundesebene)

Weiters bzw. ergänzend wird ausgeführt, dass die Umsetzung in vielen oder sogar allen Bereichen auf Schwierigkeiten stößt bzw. faktisch nicht gegeben ist oder dass es in manchen bzw. bestimmten Bereichen (Sektionen oder Regionen) gut funktioniert, in anderen jedoch nicht. Nachnominierungen werden des Öfteren eingesetzt, aber auch so kann die Frauenquote manchmal nicht erfüllt werden, da die kontrollierenden Frauengremien nicht beharrlich genug sind oder keine (geeigneten) Frauen gefunden werden können.

Vm4: Ja, in manchen Gremien haben wir es überhaupt dann nicht / oder hat es die Frauenabteilung dann auch nicht geschafft die Frauen zu finden, [...] dass sie das auch auffüllen konnte. (vida, männlich, hauptamtlich, Regionalebene, leitende Funktion)

\subsection{Ablauf der Umsetzung}

Besonders relevant ist die Quotenregelung zur Zeit der Wahlen, wenn es um die Erstellung der KandidatInnenlisten geht. Bei der Erstellung der Listen bemühen sich in der GPA-djp die für die jeweiligen Bereichen zuständigen GewerkschaftssekretärInnen, die Vorsitzenden der jeweiligen Bereiche und andere Beteiligte darum, Frauen auf die Listen zu setzen. In den Regionen sind die Frauensekretärinnen von vornherein in die Erstellung der Wahllisten involviert oder sie werden angefragt, wenn noch Frauen fehlen, oder die Frauensekretärinnen fragen von sich aus nach, wie es mit dem Frauenanteil auf den Listen aussieht.

In der vida werden zum Ablauf der Umsetzung der Frauenquote nur von wenigen Befragten konkrete Aussagen gemacht. Die Bundesfrauenvorsitzende und Frauenvorsitzenden der jeweiligen Region spielen eine wichtige Rolle bei der Umsetzung, sie achten auf die 
Einhaltung der Quote, wobei Nachnominierungen ein gängiges Mittel zu sein scheinen. Dass die Vorsitzenden der verschiedenen Bereiche auf die Einhaltung der Frauenquote achten, gibt nur eine Befragte der vida an. Ein Regionalsekretär berichtet, dass er den Beteiligten vorrechnet, wie viele Frauen zu nominieren sind, was in der Folge akzeptiert werde. Keine bzw. keiner der anderen Befragten in der vida erwähnt Aktivitäten von GewerkschaftssekretärInnen. In der GPA-djp wird deutlich häufiger über ein Engagement der Geschäftsführung und der Hauptamtlichen berichtet.

Laut Geschäftsordnung der GPA-djp muss über die Umsetzung der Quote (die auch bei den Ersatzplätzen zu erfüllen ist) regelmäßig schriftlich berichtet werden, bei Nichterreichen ist jährlich ein zusätzlicher, mündlicher Bericht abzugeben (GPA-djp, 2010). Dieser Ablauf wird auch eingehalten. Am relevantesten im Kontrollvorgang ist die Bundeskontrolle, deren Überwachung der Zusammensetzung der Gremien von den Befragten als effektiv eingeschätzt wird. Widersprüchlich sind die Angaben dazu, ob die Tätigkeit der Bundeskontrolle sich auch auf die Erstellung der Wahllisten erstreckt oder erst einsetzt, nachdem die Listen schon erstellt und die Gremien gewählt worden sind. Weniger gut hat bisher die Kontrolle zwischen den Wahlen funktioniert (wenn die Quote nicht erreicht worden war oder wenn Ausgeschiedene durch Ersatzpersonen ersetzt wurden), weil das Prozedere der Einforderung der jährlichen Berichte nicht klar geregelt war. In Bezug auf Letzteres ist zum Zeitpunkt der Befragung die Etablierung eines formalen Ablaufs geplant. Wichtig für die erfolgreiche Umsetzung in der GPA-djp ist die Unterstützung durch den Vorsitzenden und die Bundesgeschäftsführung bzw. durch die Regionalgeschäftsführungen.

Auch in der vida sollte die Kontrollkommission auf die Einhaltung der Quote schauen, dies ist aber bisher verabsäumt worden. Anders als in der GPA-djp gibt es kein festgelegtes Prozedere der Kontrolle. Ein auf der Regionalebene tätiger Befragter stellt fest, dass bei Verfehlen der Quote unklar ist, was und wie nun zu tun sei, und deshalb die Angelegenheit an die Frauenvorsitzende bzw. ein Frauengremium delegiert wird. Eine wichtige Rolle bei der Umsetzung der Frauenquote kommt den Frauenvorsitzenden der jeweiligen Region zu. Diese nominieren Frauen nach und werden zumindest in einem Bundesland vorher bereits um passende Frauen gefragt. In mehreren Bundesländern sind die Frauenvorsitzenden schwächer und die Umsetzung gelingt nicht so gut. Eine Befragte attestiert den Verantwortlichen in den Bundesländern wenig Engagement beim Auffinden von Frauen für die Gremien.

Vw3: Das Hauptproblem habe ich in einzelnen Ländern, wo es überhaupt nicht hinhaut, von der Mitgliederzahl zu den Lenkungsgremien. [...] Ich denke wirklich das resultiert daher, weil die immer schon in Funktionen waren, bestimmte Kollegen, und ja, Sesselräumen ist immer schwer, sage ich einmal. Und dann kommt natürlich der allseits beliebte Ruf, wenn man dann wirklich wem auf die Zehen steigt „Ja, wir finden ja keine Frau!“ Ja sicher, ich meine, wenn ich heute einen Namen nennen muss für ein neues Gremium, fünf alte sind mir bekannt, Männer, natürlich habe ich im Moment jetzt keine Idee, aber da muss man ja auch daran arbeiten über die Zeit. Nur auf die Idee kommen ja die Kollegen gar nicht. Es spielt auch mit sicher, weil wir keine einzige Landesgeschäftsführerin haben, das ist ausschließlich mit Männern besetzt. (vida, weiblich, hauptamtlich)

\subsection{Begleitmaßnahmen}

Um mehr Betriebsrätinnen zur Übernahme von Funktionen zu gewinnen, werden in der GPA-djp Mentoring-Programme und Bildungsmaßnahmen für Frauen durchgeführt. Für die GewerkschaftssekretärInnen wurden verpflichtende Gender-Seminare durchgeführt, um ihr diesbezügliches Bewusstsein weiterzubilden und sie (u.a.) für die Rekrutierung von Frauen 
zu schulen. Änderungen der Sitzungskultur werden als notwendig erkannt und in einem gewissen, jedoch beschränkten Ausmaß durchgeführt. Solche Maßnahmen waren bereits Teil des Quotenbeschlusses des Frauenzentralausschusses im Jahr 1996 (vgl. auch ÖGBFrauenabteilung, 1997: 28).

Auch in der vida gibt es Begleitmaßnahmen, die allerdings weniger umfangreich und formalisiert sind. Frauen werden gezielt angesprochen, um sie zur Mitarbeit in der Gewerkschaft zu ermuntern und um in der Folge über geeignete Frauen für die Umsetzung der Quote zu verfügen; in einer Region gibt es die Möglichkeit, in die Gremienarbeit der Frauenabteilung hineinzu,,schnuppern“ (Vw4). Weiters wurden zur Rekrutierung bzw. zum Halten von Frauen die Bildungsangebote angepasst.

\subsection{Wichtige Problemfelder in Zusammenhang mit der Umsetzung der Frauenquote}

\section{"Zu wenige Frauen"}

Ein in beiden Gewerkschaften häufig angeführtes Problemfeld bei der Umsetzung der Frauenquote ist die Schwierigkeit, genug Frauen für die Besetzung der Sitze in den Gremien zu finden. Die Mitglieder der gewerkschaftlichen Gremien rekrutieren sich traditionellerweise aus BetriebsrätInnen, insbesondere den Betriebsratsvorsitzenden größerer Betriebe. Betriebsratsvorsitzende sind häufig (abhängig von der Zahl der Beschäftigten im Betrieb) beruflich freigestellt und können aufgrund dessen Termine in Zusammenhang mit ihrer gewerkschaftlichen Tätigkeit leichter wahrnehmen als nicht freigestellte BetriebsrätInnen. In beiden Gewerkschaften sind - so wie in den anderen österreichischen Gewerkschaften Frauen unter den Betriebsratsvorsitzenden unterrepräsentiert, in der vida ist das Ausmaß der Unterrepräsentation stärker und betrifft auch die BetriebsrätInnen (vgl. Blaschke, 2008: 135). Zusätzlich gibt es im privaten Dienstleistungsbereich - insbesondere in den für die vida relevanten Branchen mit einem höheren ArbeiterInnenanteil - weniger Betriebsratskörperschaften (vgl. AK OÖ, 2013) und wegen des höheren Anteils an kleineren Betrieben auch weniger freigestellte Betriebsratsvorsitzende.

Der niedrige Frauenanteil bei den Betriebsratsvorsitzenden (manchmal auch bei den BetriebsrätInnen) wird als wichtiger Grund für die Schwierigkeiten genannt, genug Frauen für die Gremien zu finden. Die Unterrepräsentation von Frauen unter den Betriebsratsvorsitzenden führt in der Folge zum Problem, dass zur Erfüllung der Frauenquote Männer aus Gremien ausscheiden müssten bzw. nicht hineinkommen könnten, die als Betriebsratsvorsitzende großer Betriebe wichtig wären, unter anderem in Hinblick auf Kollektivvertragsverhandlungen. Die Belegschaften der Betriebe wählen ihre BetriebsrätInnen selbst, hier hat die Gewerkschaft formal keine Möglichkeit zu intervenieren, sie kann nur versuchen über gewerkschaftlich organisierte BetriebsrätInnen Einfluss auf die Listenerstellung zu nehmen bzw. auf die Wahl des/der Betriebsratsvorsitzenden. Konkrete diesbezügliche Aktivitäten werden allerdings nicht erwähnt.

Als Grund für die geringere Bereitschaft von Frauen eine gewerkschaftliche Funktion zu übernehmen und auch für die Unterrepräsentation von Frauen unter den Betriebsratsvorsitzenden wird am häufigsten die Belastung durch Haushalt und Familie genannt und auch die zeitliche Belastung an sich bzw. zusätzlich zur Tätigkeit als Betriebsrätin. Als ein weite- 
rer wichtiger Grund für die Schwierigkeit, Frauen für Funktionen zu finden, wird angeführt, dass Frauen zögerlicher sind, Funktionen in Betriebsrat und Gewerkschaft zu übernehmen: Männer streben solche Positionen von sich aus an und sagen rasch(er) zu, wenn sie angesprochen werden, ob sie eine Funktion übernehmen wollen. Frauen müssen angesprochen werden und auch dann überlegen sie lange und wägen sorgfältig ab, ob sie sich die Tätigkeit zutrauen und ob die damit verbundenen Verpflichtungen für sie vom Zeitaufwand her machbar sind. Dieser Unterschied im Verhalten weist auch einen Zusammenhang mit der im Durchschnitt unterschiedlichen Belastung durch die unbezahlte Haushalts- und Familienarbeit auf.

Gm1: Selbsteinschätzung, das große Problem bei der Selbsteinschätzung ist, wenn ich heute sage: Diese und jene Funktion gibt es. Also in 99 von 100 Fällen sagt mir ein Mann: Passt, das machen wir. Während es doch sehr viele Frauen gibt, die überlegen: Kann ich das? Bin ich gut genug? Die Ansprüche, die ich hätte, entspreche ich diesen Ansprüchen? Also die Selbstzweifel sind ungleich größer. (GPA-djp, männlich, hauptamtlich, Bundesebene, leitende Funktion)

Vw1: Teilweise, würde ich sagen, sind wir Frauen auch selber schuld, weil wir nicht das haben was sehr viele Männer haben, oder es haben sehr wenige Frauen das, was die Männer haben. Sie [die Frauen] kriegen etwas angeboten und denken 300 Jahre darüber nach. Ein Mann sagt: „Ich probiere es“. (vida, weiblich, ehrenamtlich, Regionalebene)

In der vida merkt eine Befragte selbstkritisch an, dass der Aufbau des weiblichen Nachwuchses vernachlässigt worden ist.

Aufgrund des Mangels an interessierten, qualifizierten und geeigneten Frauen werden die aktiven Frauen in beiden Gewerkschaften insgesamt stark eingesetzt. Entsprechend dem stärkeren Mangel an verfügbaren Frauen in der vida wird die starke Belastung der aktiven Frauen dort häufiger genannt als in der GPA-djp und es wird mehrmals angeführt, dass dadurch die Gefahr des „Ausbrennens“ bzw. der Überforderung besteht, was dann zum Ausscheiden oder zum Rückzug der betreffenden Frauen führen kann.

Vw8: Ja, aber sie werden dann einfach verheizt. Genauso ist es. Man sieht ein Potenzial, der hängt man 17.000 Amteln um. [...] Und somit wird man dann sehr schnell verheizt und dann gibt man halt als Frau sehr leicht w.o. Also wir haben in den letzten vier Jahren haben wir wirklich Spitzenbetriebsrätinnen, ich will nicht sagen verloren, aber die aus diversen Gremien, aus wichtigen Gremien, rausgegangen sind und gesagt haben „Stop“. (vida, weiblich, ehrenamtlich, Bundes- und Regionalebene)

Hier wird eine schwer zu überwindende Dynamik geschildert: Die Überlastung der wenigen aktiven Frauen führt dazu, dass der Mangel an aktiven Frauen verstärkt wird.

\section{Männlicher Widerstand}

In beiden Gewerkschaften wird angegeben, dass Widerstand seitens männlicher Gewerkschafter die Umsetzung der Quote erschwert, in der GPA-djp allerdings deutlich seltener als in der vida (dies steht in Widerspruch zu einem früheren Befund, jedoch war dort die empirische Basis deutlich schmäler - Blaschke, 2008: 162). Dieser Widerstand drückt sich vor allem darin aus, dass zu wenig für die Umsetzung der Frauenquote getan wird bzw. Frauen nicht gefördert werden (letzteres wird in der vida deutlich häufiger beobachtet als in der GPA-djp); teilweise werden diese Verhaltensweisen explizit älteren Männern zugeschrieben. In der vida lassen zudem mehrere Aussagen auf eine massivere Ausformung der männlichen Opposition zur Quotenregelung schließen, bei der es zur Behinderung von 
Frauen durch Männer kommt. Es kann dort auch eine Form des Widerstands identifiziert werden, bei der die Quote ,nur der Form halber“ (Vm8) erfüllt wird: Es werden ungeeignete Frauen nominiert bzw. Frauen, denen nur die Rolle der Quotenfrau zugedacht ist, oder die Quote wird nur mit Nachnominierungen und Berücksichtigung der Ersatzplätze erfüllt.

Männlicher Widerstand entsteht in beiden Gewerkschaften, weil Männer zur Erfüllung der Frauenquote ihre Positionen räumen müssten oder dies zumindest befürchten. In der vida wird dies deutlich häufiger genannt und es wird dort des Weiteren angeführt, dass Männer freiwerdende Positionen weiterhin über ihre männlichen Netzwerke besetzen wollen. Auch in der GPA-djp ist es durch die Einführung der Frauenquote dazu gekommen, dass bisher durch Männer besetzte Mandate an Frauen gegangen sind, dieser Umstellungsprozess scheint aber in der GPA-djp schneller abgeschlossen worden zu sein als in der vida. Die noch andauernde Verringerung der Mandate nach der Fusion verstärkt in der vida die Problematik, dass zur Erfüllung der Frauenquote Männer ihre Positionen räumen müssen, und dies könnte dazu beitragen, dass männlicher Widerstand häufiger als Problem genannt wird als in der GPA-djp.

In der vida werden Frauen auch dadurch ausgebremst, dass sie härter arbeiten müssen, um akzeptiert zu werden, und strenger beurteilt werden als Männer. Dies führt dann teilweise dazu, dass Frauen ihr gewerkschaftliches Engagement aufgeben.

Vw8: Du musst es dir wirklich beinhart erkämpfen. Wo es als Mann dir manchmal in den Schoß gelegt wird, [...] also eine Frau muss dreifach so viel arbeiten[...], das muss man sich wirklich hart erarbeiten als Frau und das ist halt eine lange Durststrecke, die nicht jede Frau schafft. (vida, weiblich, ehrenamtlich, Bundes- und Regionalebene)

\section{"Quotenfrauen"}

Mit „Quotenfrau“ wird eine Frau bezeichnet, die aufgrund der Frauenquote in ein Gremium gewählt oder nominiert worden ist. Meist wird der Begriff abwertend verwendet und drückt aus, dass die betreffende Frau weder qualifiziert noch engagiert ist.

Dass es vorkommt, dass zur Erfüllung der Quote nominierte Frauen dann nicht zu den Sitzungen kommen, wird in beiden Gewerkschaften von einigen Befragten angegeben. Die betreffenden Befragten der GPA-djp weisen gleichzeitig darauf hin, dass dieses Verhalten auch bei Männern zu beobachten ist, bei ihnen aber (im Gegensatz zu den Frauen) nicht negativ auffällt. In der vida wird angemerkt, dass es Frauen, insbesondere denen, die nicht freigestellte Betriebsrätin sind, oft nicht möglich ist, zu den Sitzungen zu kommen. Dass zur Erfüllung der Quote auch Frauen nominiert werden, die nicht qualifiziert sind oder die man gar nicht gefragt hat, wird in der GPA-djp nur von zwei Befragten und nur für Einzelfälle beobachtet. In der vida wird die Nominierung unqualifizierter und unengagierter Frauen etwas häufiger und nicht als Einzelfallphänomen geschildert.

Eine explizite Abwertung der in den Gremien tätigen Frauen als „Quotenfrauen“ wird in der GPA-djp lediglich von einer Befragten für die erste Phase nach der Einführung berichtet. Für die aktuelle Situation wird sie nur in der vida genannt und dies von mehreren Befragten.

Vm5: Also der Nachteil ist in meinen Augen, dass es schnell einmal heißt: „Okay, das ist jetzt die Quotenfrau.“ Dass dadurch die Qualifikation abgesprochen wird, aufgrund der Quote. Was ich schon auch erlebt habe und was halt zum Teil sehr schade ist, wenn man wo eine sehr engagierte gute Frau hat und dann wird ihr nachgesagt, 
okay, sie ist es aber nur wegen der Quote. Und das dann auch immer schwer ist, weil klar, die Nominierung hat dann aufgrund der Quote vielleicht auch so stattgefunden, aber natürlich auch aufgrund der Qualifikation. (vida, männlich, hauptamtlich, Regionalebene)

Eine Befragte der vida führt aus, dass in ihrer Region von den Frauen, die zur Erfüllung der Quote in ein Gremium nominiert oder gewählt sind, sogar erwartet wird, dass sie sich nicht einbringen. Ähnlich wie in der GPA-djp, wo das Fernbleiben bei Frauen stärker auffällt als bei Männern, führen Befragte in der vida an, dass die Frauen in den Gremien kritischer beurteilt werden als Männer und in der Folge als „Quotenfrau“ abqualifiziert werden.

\section{Diskussion}

\section{Implementation der Frauenquote in der GPA-djp und in der vida}

In beiden Gewerkschaften wurde die Frauenquote im Zuge organisatorischer Veränderungen als Teil einer neuen Geschäftsordnung beschlossen. So wie in anderen Fällen der Einführung von Quotenregelungen (vgl. oben) ging sie auch hier auf die Forderung von Frauen zurück und war der Nutzen für die Organisation wesentliches Motiv auf Seiten männlicher Entscheidungsträger, im Fall der vida auch der Druck aufgrund des bevorstehenden Quotenbeschlusses des ÖGB. Im Gegensatz zur vida ging dem Beschluss in der GPA ein längerer, umfassender Diskussionsprozess voraus.

Begleitmaßnahmen zur erfolgreichen Implementation der Quote sind von beiden Gewerkschaften ergriffen worden, wobei diese in der GPA-djp vielfältiger gestaltet sind. So sind Regelungen zum Ablauf der Umsetzung und Aktivitäten zur Kontrolle (Berichtspflichten, Interventionen seitens der Geschäftsführung) in der GPA-djp besser ausgebaut als in der vida. Reformen der Organisationskultur als Begleitmaßnahme zur Quote werden nur in der GPA-djp angegangen, dies allerdings lediglich in einem beschränkten Rahmen: Zum einen wurden in bestimmten Gremien Änderungen der Sitzungskultur vorgenommen, zum anderen gab es Gender-Schulungen für alle Hauptamtlichen, die über Bewusstseinsbildung zu einer Änderung der Gewerkschaftskultur führen können. Andere von beiden Gewerkschaften ergriffene Begleitmaßnahmen richten sich direkt an Frauen, hierzu zählen die persönliche Ansprache und spezifische bzw. angepasste Bildungsangebote; beide sind in der GPA-djp umfassender ausgestaltet, zusätzlich gibt es dort Mentoring-Programme.

Die Umsetzung und Erfüllung der Frauenquote funktioniert zum Erhebungszeitpunkt (2014) in der GPA-djp besser als in der vida: nur in wenigen Ausnahmefällen wird die Quote in der GPA-djp nicht erfüllt, in der vida hingegen kommt dies öfters vor. Der Zeitraum, seit dem die Quotenregelung gilt, ist in der vida mit acht Jahren kürzer als in der GPA-djp mit 17 Jahren. Auch in der GPA-djp werden für die ersten Jahre mehrere Probleme beim Erreichen der Quote berichtet. Allerdings war in der GPA-djp zum vergleichbaren Zeitpunkt nach Beschluss der Quotenregelung (d.h. acht Jahre danach) die Frauenquote in stärkerem Ausmaß umgesetzt als in der vida. Die Übergangsphase bis zur vollständigen Erfüllung der Frauenquote dauert in der vida somit länger als in der GPA-djp; die anhaltende Verringerung der Gremiengrößen und damit der Zahl der Mandate nach der Fusion trägt dazu vermutlich bei. 
Dass es im Vorfeld der Einführung der Frauenquote keinen breiten Diskussionsprozess gab, in dem es möglich gewesen wäre, SkeptikerInnen mit Argumenten zu überzeugen, könnte ein Grund für die weniger zufriedenstellende Umsetzung in der vida sein. Ein zweiter Grund für die niedrigere Effektivität der Frauenquote in der vida dürfte das geringere Ausmaß an Begleitaktivitäten sein. Der Geschäftsführung und den Hauptamtlichen der vida, insbesondere den Männern unter ihnen, scheint die Umsetzung der Frauenquote ein geringeres Anliegen zu sein als in der GPA-djp. Dies könnte mit einem niedrigeren Grad an Überzeugung (u.a. aufgrund des Fehlens eines breiten Diskussionsprozesses) zu tun haben.

Der Befund, dass Begleitmaßnahmen, insbesondere wirksame Kontrollinstrumente, eine erfolgreiche Umsetzung der Frauenquote fördern, steht im Einklang mit den oben angeführten Schlussfolgerungen von Bothfeld \& Rouault (2015) und Matland (2006). Die Ergebnisse zu hemmenden Umständen des Ablaufs von Einführung und Implementation, decken sich mit Ergebnissen zu Gleichstellungsmaßnahmen in Betrieben. Auch dort behinderte das Ausbleiben eines intensiveren internen Diskussionsprozesses die Implementation und eine unzureichende Unterstützung der Frauenförderung durch die Geschäftsführung führte dazu, dass - vorwiegend männliche - Beschäftigte verschiedener Hierarchieebenen die Umsetzung blockierten (May, 1998: 83; Riegraf, 1996: 210-213).

Die Erfüllung der Frauenquote stößt in beiden Gewerkschaften auf das Problem, dass es teilweise schwierig ist, Frauen für die nun zu besetzenden Funktionen zu finden. In der GPA-djp wird dies allerdings seltener angegeben als in der vida. Der Mangel an verfügbaren Frauen führt in der vida deutlich häufiger als in der GPA-djp dazu, dass Nachnominierungen notwendig sind; und anders als in der GPA-djp werden dann in der vida manchmal ungeeignete Frauen nominiert oder die Quote kann nicht erfüllt werden. Ein Grund für den Unterschied zur GPA-djp könnte die geringere Dichte an Betriebsratskörperschaften im privaten Dienstleistungssektor sein, die die Rekrutierung von Funktionärinnen erschwert. Ein zweiter wesentlicher Grund für den Mangel an weiblichen Funktionären in der vida dürfte die weniger intensive Nachwuchsförderung (eine wichtige Begleitmaßnahme zur Umsetzung der Frauenquote) sein. Eine Parallele besteht zwischen dem in der vida immer wieder genannten Mangel an Funktionärinnen und den Schwierigkeiten, Gesprächspartnerinnen für das Forschungsprojekt zu finden. Infolge des Mangels an geeigneten oder interessierten Frauen kommt es zu einer Überlastung derjenigen Frauen, die aktiv sind. Analoges wird für die Schweizer Gewerkschaft Unia berichtet (wo die Situation dadurch verschärft wird, dass die Frauenquote über dem Frauenanteil an den Mitgliedern liegt (Monney, Fillieule \& Avanza, 2013: 45-49).

\section{Gründe für die Unterschiede zwischen GPA-djp und vida}

Die geringere Häufigkeit an Betriebsratskörperschaften (insbesondere solcher mit freigestellten Vorsitzenden) in den für die vida relevanten Branchen des privaten Dienstleistungsbereichs bietet eine Erklärung dafür, dass Frauen in der vida weniger stark an den Entscheidungsstrukturen teilhaben als in der GPA-djp. Im Rahmen der vor der Erhebung formulierten Annahmen zu den Gründen für die geringere Partizipation auf Seiten der Frauen in der vida stellt dieser Faktor eine Facette der Arbeitsbedingungen dar. Abseits dessen finden die Annahmen im empirischen Material weder Bestätigung noch Widerlegung, hierzu wären noch weitere Untersuchungen notwendig. 
In Bezug auf die Männer unterstützen die Ergebnisse die Annahme, dass Männer mit höherem Qualifikationsniveau Frauen eher in die Gewerkschaft integrieren. Männer, auch Männer in höheren Positionen, werden in der GPA-djp deutlich häufiger als unterstützend genannt als in der vida. Zudem wird in der vida öfter als in der GPA-djp davon berichtet, dass Männer die Umsetzung der Frauenquote behindern. Überwiegend wirkt der Nutzen für die Gewerkschaft als treibende Kraft hinter der Unterstützung der GPA-djp-Männer, manchmal auch normativer Druck (ausgeübt durch die Geschäftsführung) oder persönliche Überzeugung. In der vida scheint normativer Druck („Die Frauenquote ist zu erfüllen.“) das Verhalten der Männer dort, wo sie die Umsetzung der Frauenquote unterstützen oder nicht behindern, stärker zu bestimmen als in der GPA-djp.

Die Unterschiede in der Gestaltung des Einführungsprozesses deuten auf Unterschiede in der Gewerkschaftskultur hin: Aus dem empirischen Material lässt sich schließen, dass die GPA bzw. GPA-djp als Angestelltengewerkschaft den Anspruch hat, Maßnahmen zu diskutieren und Akzeptanz herbeizuführen, während in der ArbeiterInnengewerkschaft vida die Führung - im Vergleich zur GPA-djp - stärker vorgibt, was zu geschehen hat. Die Spezifika der jeweiligen Gewerkschaftskultur wirken sich in der Folge auf das Verhalten der AkteurInnen aus. Ob und inwieweit die Unterschiede in der Gewerkschaftskultur von GPAdjp und vida mit dem Qualifikationsniveau und dem Angestelltenstatus in Zusammenhang stehen, wäre anhand von Untersuchungen weiterer Gewerkschaften zu klären; ebenso ob sich die beobachtete Verhaltensdifferenz zwischen unterschiedlich hoch gebildeten Männern verallgemeinern lässt.

Die Unterschiede zwischen GPA-djp und vida sind durch zwei Faktoren außerhalb der jeweiligen Gewerkschaften mitbedingt. Dies betrifft zum einen die Vorgabe durch die Frauenquote des ÖGB, welche die Spezifika des Prozesses der Einführung und in der Folge auch der Umsetzung in der vida beeinflusste, und zum anderen die geringere Zahl an Betriebsratskörperschaften in den relevanten Branchen des privaten Dienstleistungsbereichs. Zukünftige Studien in Gewerkschaften mit teils gleichen und teils differierenden externen Rahmenbedingungen würden mehr Aussagen über die Wirkung einzelner Faktoren erlauben.

\section{Wege zur gleichberechtigten Teilhabe von Frauen}

Die Faktoren auf Makro-, Meso- und Mikroebene, die zuvor zur Unterrepräsentation von Frauen geführt haben, werden durch die Quote nicht zum Verschwinden gebracht und können in der Folge die Umsetzung behindern. Dies äußert sich dann z.B. darin, dass es schwierig ist, geeignete Frauen für die Umsetzung der Quote zu finden, oder dass durch Strategien seitens männlicher Gewerkschafter die Quote unterlaufen wird. Deshalb sind für eine erfolgreiche Umsetzung Begleitmaßnahmen notwendig.

Die primäre Zuständigkeit der Frauen für die private Versorgungsarbeit stellt ein wesentliches Hindernis für die gleichberechtigte Teilhabe von Frauen dar, nicht nur in den Gewerkschaften, sondern auch in anderen gesellschaftlichen Bereichen. Eine geschlechtergerechte Verteilung der Versorgungsaufgaben würde es berufstätigen Frauen erleichtern, sich in Betriebsrat und Gewerkschaft zu engagieren. Die auf der Makroebene angesiedelte innerfamiliale Arbeitsteilung wird von den beiden untersuchten Gewerkschaften (ebenso wie von den österreichischen Gewerkschaften generell) jedoch nicht als relevantes Handlungsfeld gesehen; 
es werden seitens der Gewerkschaften kaum Aktivitäten gesetzt, um Männer stärker in die häusliche Versorgungsarbeit einzubinden (vgl. Sorger, 2014: 87, 251).

Ähnlich wird die Unterrepräsentation der Frauen unter den Betriebsratsvorsitzenden (die die Umsetzung der Frauenquote erschwert) als von Gewerkschaftsseite her nur wenig beeinflussbar wahrgenommen. Eine gesetzliche Regelung analog der deutschen Geschlechterquote für die Betriebsratskörperschaften wird in Österreich derzeit nicht diskutiert. Es ist auch fraglich, ob sie in den beiden untersuchten Gewerkschaften zu einer Vergrößerung des weiblichen Reservoirs für gewerkschaftliche Gremien führen würde. Denn die männliche Vorherrschaft bei den Vorsitzenden würde voraussichtlich weiter bestehen, und in den weiblich dominierten Branchen wäre keine Steigerung des Frauenanteils in den Betriebsratskörperschaften zu erwarten.

Während die Möglichkeiten des Einflusses auf externe Faktoren, die zu Geschlechterungleichheiten führen, für die Gewerkschaften begrenzt sind oder als begrenzt wahrgenommen werden, unterliegen Gewerkschaftsstruktur und -kultur (die Mesoebene) in stärkerem Ausmaß einer gezielten Gestaltbarkeit (auch die Frauenquote ist der Mesoebene zugehörig). Die beiden untersuchten Gewerkschaften konzentrieren sich hier auf Maßnahmen zur Umsetzung und Kontrolle der Quote. Eine Änderung der männlich geprägten Organisationskultur wird jedoch von mehreren AutorInnen als notwendig für eine gleichberechtigte Teilhabe von Frauen angesehen, nicht nur in Gewerkschaften, sondern auch in anderen Organisationen (z.B. Krell, 2010: 477; Ledwith, 2012; Morgenroth, 1996: 197-199). Beispielsweise könnten Reformen der Gremienarbeit und Sitzungskultur, die den zeitlichen Restriktionen berufstätiger Frauen mit Kindern Rechnung tragen, eine Möglichkeit sein, die Beteiligung von Frauen zu erhöhen und die Belastung der aktiven Frauen zu verringern. Solche Reformen der Organisationskultur werden in den beiden untersuchten Gewerkschaften bislang nur in der GPA-djp angegangen, allerdings lediglich in einem beschränkten Rahmen. Maßnahmen zur Bewusstseinsbildung, wie sie von der GPA-djp (neben anderem) zur Änderung der Gewerkschaftskultur eingesetzt werden, können auch einen Beitrag zum Abbau von auf der Handlungsebene wirksamen Geschlechtsstereotypen leisten, ebenso wie spezifische unterstützende Angebote für Frauen.

\section{Literatur}

Abels, G., \& Behrens, M. (2002). ExpertInnen-Interviews in der Politikwissenschaft. Geschlechtertheoretische und politikfeldanalytische Reflexion einer Methode. In A. Bogner, B. Littig, \& W. Menz (Hrsg.), Das Experteninterview. Theorie, Methode, Anwendung (S. 173-190). Opladen: Leske + Budrich. doi: 10.1007/978-3-322-93270-9_8

AK OÖ (Arbeiterkammer Oberösterreich) (2013). Besser mit Betriebsrat. Abgerufen von http://ooe.arbeiterkammer.at/beratung/arbeitundgesundheit/arbeitsklima/arbeitsklima_index/Arbe itsklima_Index_November_2013.html

Behnke, C., \& Meuser, M. (1999). Geschlechterforschung und qualitative Methoden. Opladen: Leske + Budrich. doi: 10.1007/978-3-322-97403-7

Berger, U., \& Bernhard-Mehlich, I. (1999). Die Verhaltenswissenschaftliche Entscheidungstheorie. In A. Kieser (Hrsg.), Organisationstheorien (S. 133-168). Stuttgart, Berlin, Köln: Kohlhammer.

Blaschke, S. (2008). Frauen in Gewerkschaften. Zur Situation in Österreich und Deutschland aus organisationssoziologischer Perspektive. München und Mering: Hampp. 
Blaschke, S. (2011). Frauen in Gewerkschaften. Zur Situation in Österreich und Deutschland aus organisationssoziologischer Perspektive. Unveröffentlichte Habilitationsschrift an der Universität Wien. Wien.

Blaschke, S. (2015). Female representation in the decision-making structures of trade unions: The influences of sector, status and qualification. Journal of Industrial Relations, 57, 726-747. doi: $10.1177 / 0022185615590904$

Bogner, A., Littig, B., \& Menz, W. (2014). Interviews mit Experten. Eine praxisorientierte Einführung. Wiesbaden: VS.

Bothfeld, S., Hübers, S., \& Rouault, S. (2010). Gleichstellungspolitische Rahmenbedingungen für das betriebliche Handeln. Ein internationaler Vergleich. In Projektgruppe GiB (Hrsg.), Geschlechterungleichheiten im Betrieb. Arbeit, Entlohnung und Gleichstellung in der Privatwirtschaft (S. 21-88). Berlin: edition sigma. doi: 10.5771/9783845266565-21

Bothfeld, S., \& Rouault, S. (2015). Was macht eine effiziente Gleichstellungspolitik aus? Das Instrument Frauenquote im internationalen Vergleich. WSI Mitteilungen, 68 (1), 25-34.

Cockburn, C. (1991). In the Way of Women. Men's Resistance to Sex Equality in Organizations. Basingstoke: Macmillan. doi: 10.1007/978-1-349-21571-3

Colgan, F., \& Ledwith, S. (2000). Diversity, Identities and Strategies of Women Trade Union Activists. Gender, Work and Organization, 7, 242-257. doi: 10.1111/1468-0432.00112

Colgan, F., \& Ledwith, S. (2002). Gender and diversity. Reshaping union democracy. Employee Relations, 24, 167-189. doi: 10.1108/01425450210420901

Dahlerup, D. (Hrsg.) (2006a). Women, Quotas and Politics. London: Routledge.

Dahlerup, D. (2006b). Conclusion. In D. Dahlerup (Hrsg.), Women, Quotas and Politics (S. 293-307). London: Routledge.

Dickens, L. (2000). Collective bargaining and the promotion of gender equality at work: opportunities and challenges for trade unions. Transfer, 6, 193-208. doi: 10.1177/102425890000600205

Diekmann, A. (2012). Empirische Sozialforschung. Grundlagen, Methoden, Anwendungen. Reinbek bei Hamburg: Rowohlt.

Frauenbericht 2010. Bericht betreffend die Situation von Frauen in Österreich im Zeitraum von 1998 bis 2008. Hrsg. von der Bundesministerin für Frauen und Öffentlichen Dienst im Bundeskanzleramt Österreich. Wien: Bundeskanzleramt.

Funder, M. (2002). Feministische Theorien. In T. Bonacker (Hrsg.): Sozialwissenschaftliche Konflikttheorien (S. 293-318). Opladen: Leske + Budrich. doi: 10.1007/978-3-322-94989-9_15

Gewerkschaft Bau-Holz (2010). Geschäftsordnung der Gewerkschaft Bau-Holz, beschlossen am 18. Gewerkschaftstag der Gewerkschaft Bau-Holz 24.-25. November 2010.

GPA (Gewerkschaft der Privatangestellten) (1998). Geschäfts- und Wahlordnung, beschlossen am Gewerkschaftstag 1998. O.O.

GPA (Gewerkschaft der Privatangestellten) (2000). Geschäfts- und Wahlordnung, beschlossen am Sondergewerkschaftstag 2000. O.O.

GPA-djp (Gewerkschaft der Privatangestellten, Druck, Journalismus, Papier) (2010). Geschäfts- und Wahlordnung, beschlossen am Bundesforum 2010. O.O.

Healy, G., \& Kirton, G. (2000). Women, Power and Trade Union Government in the UK. British Journal of Industrial Relations, 38, 343-360. doi: 10.1111/1467-8543.00168

HGPD (Gewerkschaft Hotel, Gastgewerbe, Persönliche Dienste) (1995). Geschäftsordnung. Wien.

Hoecker, B. (1995). Politische Partizipation von Frauen. Kontinuität und Wandel des Geschlechterverhältnisses in der Politik. Ein einführendes Studienbuch. Opladen: Leske + Budrich. doi: 10.1007/978-3-322-95761-0 
Inhetveen, K. (2002). Institutionelle Innovation in politischen Parteien. Geschlechterquoten in Deutschland und Norwegen. Opladen: Leske + Budrich. doi: 10.1007/978-3-663-07909-5

Jarosch, M. (2001). Frauenquoten in Österreich. Grundlagen und Diskussion. Innsbruck u.a.: Studienverlag

Kassel, B. (2001). ...letztlich ging es doch voran! Zur Frauenpolitik der Gewerkschaft ÖTV 19491989. o. O.: ver.di.

Kirsch, A., \& Blaschke, S. (2014). Women's quotas and their effects: A comparison of Austrian and German trade unions. European Journal of Industrial Relations, 20, 201-217. doi: $0.1177 / 0959680113518231$

Kirton, G. (2005). The influences on women joining and participating in unions. Industrial Relations Journal, 36, 386-401. doi: 10.1111/j.1468-2338.2005.00366.x

Kirton, G., \& Healy, G. (2004). Shaping Union and Gender Indentities: A Case Study of WomenOnly Trade Union Courses. British Journal of Industrial Relations, 42, 303-323. doi: 10.1111/j.1467-8543.2004.00316.x

Koppetsch, C., \& Burkart, G. (1999). Die Illusion der Emanzipation. Zur Wirksamkeit latenter Geschlechtsnormen im Milieuvergleich. Konstanz: UVK.

Krell, G. (2010). Führungspositionen. In Projektgruppe GiB (Hrsg.), Geschlechterungleichheiten im Betrieb. Arbeit, Entlohnung und Gleichstellung in der Privatwirtschaft (S. 423-484). Berlin: edition sigma.

Krook, M. L. (2008). Quota Laws for Women in Politics: Implications for Feminist Practice. Social Politics, 15, 345-368. doi: 10.1093/sp/jxn014

Kuckartz, U. (2012). Qualitative Inhaltsanalyse. Methoden, Praxis, Computerunterstützung. Weinheim und Basel: Beltz Juventa.

Ledwith, S. (2012). Gender politics in trade unions. The representation of women between exclusion and inclusion. Transfer, 18, 185-199. doi: 10.1177/1024258912439145

Lippert, S. (2002). Politisches Engagement - noch immer eine Frage des Geschlechts? Geschlechtsspezifische Aspekte politischer Partizipation unter besonderer Berücksichtigung politischer Proteste. Hamburg: Kovac.

Littig, B. (2002). Interviews mit Experten und Expertinnen. Überlegungen aus geschlechtertheoretischer Sicht. In A. Bogner, B. Littig, \& W. Menz (Hrsg.), Das Experteninterview. Theorie, Methode, Anwendung (S. 191-206). Opladen: Leske + Budrich. doi: 10.1007/978-3-322-93270-9_9

Matland, R. E. (2006). Electoral quotas. Frequency and effectiveness. In D. Dahlerup (Hrsg.), Women, Quotas and Politics (S. 275-292). London: Routledge.

May, B. (1998). Unterstützende und hemmende Faktoren bei der Entwicklung und Einführung von betrieblichen Frauenförderungsmaßnahmen. In R. Bendl, U. Papouschek, \& U. Pastner (Hrsg.), Im Aufbruch. Betriebliche Frauenförderung in Österreich (S. 71-88). Frankfurt am Main: Peter Lang.

Mayring, P. (2003). Qualitative Inhaltsanalyse. Grundlagen und Techniken. Weinheim: Beltz.

McBride, A. (2001). Gender Democracy in Trade Unions. Aldershot: Ashgate.

Meuser, M., \& Nagel, U. (1991). ExpertInneninterviews - vielfach erprobt, wenig bedacht. Ein Beitrag zur qualitativen Methodendiskussion. In D. Garz, \& K. Kraimer (Hrsg.), Qualitativ-empirische Sozialforschung. Konzepte, Methoden, Analysen (S. 441-471). Opladen: Westdeutscher Verlag.

Meyer, J. W., \& Rown, B. (1977). Institutionalized Organizations: Formal Structures as Myth and Ceremony. American Journal of Sociology, 83, 340-363. doi: 10.1086/226550

Monney, V., Fillieule, O., \& Avanza, M. (2013). Les souffrances de la femme-quota. Le cas du syndicat suisse Unia. Travail, genre et sociétés, (30), 33-51. doi: 10.3917/tgs.030.0033 
Morgenroth, C. (1996): Die engagierte Frau. Frauen und Interessenorganisationen. Münster: Westfälisches Dampfboot.

Neyer, G. (1995). Frauen und Gewerkschaften. In Frauenbericht 1995. Bericht über die Situation der Frauen in Österreich (S. 614-618). Hrsg. von der Bundesministerin für Frauenangelegenheiten im Bundeskanzleramt. Wien Bundeskanzleramt.

Plogstedt, S. (2015). Mit vereinten Kräften. Die Gleichstellungsarbeit der DGB-Frauen in Ost und West (1990-2010). Gießen: Psychosozial-Verlag.

ÖGB-Frauenabteilung (1997). Tätigkeitsbericht der ÖGB-Frauenabteilung 1996. Wien: ÖGB.

Pfarr, H. M., \& Bertelsmann, K. (1989). Diskriminierung im Erwerbsleben. Ungleichbehandlungen von Frauen und Männern in der Bundesrepublik Deutschland. Baden-Baden: Nomos.

Ridgeway, C. L., \& Correll, S. J. (2004). Unpacking the Gender System. A Theoretical Perspective on Gender Beliefs and Social Relations. Gender \& Society, 18, 510-531. doi: 10.1177/0891243204265269

Riegraf, B. (1996). Geschlecht und Mikropolitik. Das Beispiel betrieblicher Gleichstellung. Opladen: Leske + Budrich. doi: 10.1007/978-3-322-95744-3

Schreier, M. (2012). Qualitative Content Analysis in Practice. London: Sage.

Schulz, W., \& Hummer, C. (2005). Veränderungen des Zusammenlebens und Wandel der Einstellungen zu Ehe und Familie. In W. Schulz, M. Haller, \& A. Grausgruber (Hrsg.), Österreich zur Jahrhundertwende. Gesellschaftliche Werthaltungen und Lebensqualität 1986-2004 (S. 343366). Wiesbaden: VS. doi: 10.1007/978-3-322-80738-0_11

Sebinger, S., \& Nemella, J. (2005). Parteipräferenz und politische Partizipation. In W. Schulz, M. Haller, \& A. Grausgruber (Hrsg.), Österreich zur Jahrhundertwende. Gesellschaftliche Werthaltungen und Lebensqualität 1986-2004 (S. 433-460). Wiesbaden: VS. doi: 10.1007/978-3-32280738-0_14

Sorger, C. (2014). Wer dreht an der Uhr? Geschlechtergerechtigkeit und gewerkschaftliche Arbeitszeitpolitik. Münster: Westfälisches Dampfboot.

Terry, M. (1996). Negotiating the Government of Unison: Union Democracy in Theory and Practice. British Journal of Industrial Relations, 34, 87-110. doi: 10.1111/j.1467-8543.1996.tb00472.x

Thompson, J. D. (1967): Organizations in Action: Social Science Bases of Adminstrative Theory. New York: McGraw-Hill.

Trebilcock, A. (1991): Strategies for strengthening women's participation in trade union leadership. International Labour Review, 130, 407-426.

vida (2006): Statuten. Wien: vida.

Wetterer, A. (2006): Ordentlich in Unordnung. Widersprüche im sozialen Wandel der Geschlechterverhältnisse. Österreichische Zeitschrift für Soziologie, 31, 5-22. doi: 10.1007/s11614-006-0076-8 
DuEPublico

Duisburg-Essen Publications online

Dieser Text wird über DuEPublico, dem Dokumenten- und Publikationsserver der Universität Duisburg-Essen, zur Verfügung gestellt. Die hier veröffentlichte Version der EPublikation kann von einer eventuell ebenfalls veröffentlichten Verlagsversion abweichen.

DOI: $\quad$ 10.3224/indbez.v24i1.05

URN: urn:nbn:de:hbz:464-20200717-125927-5

Dieses Werk kann unter einer Creative Commons Namensnennung - Weitergabe unter gleichen Bedingungen 4.0 Lizenz (CC BY-S 4.0) genutzt werden. 